\title{
Difficult hysterectomy - a nightmare for gynaecologists
}

\author{
Prof (Dr) Hem Kanta Sarma
}

\author{
Correspondence: Prof (Dr) Hem Kanta Sarma, Professor \& HOD, Deptt. of Obstetrics \& \\ Gynaecology, Jorhat Medical College, Jorhat, Assam, India; \\ Email - sarmahemkanta@gmail.com
}

Distributed under Attribution-NonCommercial-ShareAlike 4.0 International (CC BY-NC-SA 4.0)

Hysterectomy is one of the most commonly performed major gynecological procedures worldwide. Out of 6 , 00,000 hysterectomies performed in a year in USA $60 \%$ are performed abdominaly ${ }^{1}$. More and more hysterectomies are being done by laparoscopic or Robotic procedures now a day. Recent graduates in gynaecology are less interested to perform vaginal hysterectomy resulting in a decreasing trend of vaginal hysterectomy ${ }^{2}$.

Pelvic anatomy is distorted by large fibroids, severe endometriosis, post operative adhesions and morbid obesity making the surgery difficult. Those patients who come for radical surgery for malignancy especially carcinoma cervix after receiving primary radiotherapy sometime shows extensive adhesions and fibrosis of the pelvic organs making the anatomy distorted and occasionally obscured. In these circumstances, many a time the surgeon has to abandon the process or cut short the procedure by doing a debulking surgery or a subtotal hysterectomy. Sometime in the process of doing a difficult hysterectomy injury of the adjacent organs may occur. Occasionally a vascular damage may occur to these structures leading to fistula formation postoperatively. In some cases ureteral obstruction may lead to hydronephrosis. To avoid all these an experienced hand is necessary. A good understanding of the pelvic anatomy and expected pathological changes of the organs involved is a pre requisite. A thorough evaluation of the patient and the disease with proper knowledge of extension of the disease with possible involvement of the surrounding viscera is a must.

Efforts of developing standard techniques for dealing with such difficult hysterectomies have been going on in different parts of the world. T Lee and his team developed such techniques and used specific rules to obtain the best result with safety. They used different approaches in different situations ${ }^{3}$, viz, anterior approach for big fibroids, posterior approach for adhesions and lateral approach for endometriosis.

\section{Difficult vaginal hysterectomy}

To deal with these situations the following five points are key to success ${ }^{4}$ -

1. Surgical experience

2. Adequate exposure

3. Entry in to anterior cul de sac

4. Uterine mobility

5. Good morcellation technique

Chen and Colleagues developed and validated the vaginal surgical skill index (VSSI) with 13 aspects of successful vaginal surgery ${ }^{5}$. In vaginal hysterectomy inadvertent bladder injury is likely in difficult cases. To avert such injuries the following maneuvers are useful -

1. Trendelenburgh position ( author's personal experience )

2. Stay on uterus. Stay deep and cut into the uterus for difficult fibrotic adhesions

3. Retrograde filling of bladder with normal saline or dye may help in defining the anatomy

4. Postpone anterior entry. Entry through the posterior fornix (i.e, POD) is done first. Anterior pouch is opened only after the cardinal ligaments are divided.

5. Uterine sound is put in urinary bladder to see the extent of it. 
6. In case of small uterus, a finger through the posterior opening hooks the uterine fundus and lift the peritoneum of anterior pouch before opening.

\section{Difficult abdominal hysterectomy}

Post radiation therapy patients of carcinoma cervix when comes for radical hysterectomy sometimes present as frozen pelvis with almost complete obliteration of the pelvic cavity where the uterus and appendages are obscured. A very careful dissection is needed with proper visualization and palpation of the structures. The approach should always be through the easiest waypreferably around the uterus or ovaries. In patients with parametrial infiltration where ureteral mobilization is difficult. Ureter may be divided and reimplanted in the urinary bladder. To avoid the ureteric injury where it is anticipated, a uroglow catheter or a ureteric catheter with infra-red illumination can be kept in place before the procedure. It will help in dissection avoiding uretric damage.

In case of big uterine myoma the following facts are useful -

1. In case of anterior myoma, with the help of a myoma screw the uterus is to be elevated to keep away from the urinary bladder.

2. If the myoma is obscuring the structures to be clamped or cut, a myomectomy may be performed as a first step followed by clamping of the pedicles.

Post caesarean scar adhesions with urinary bladder are a difficult situation at times. In this case with the guidance of the folly's catheter in the bladder a lateral approach from sides will be useful. Extensive adhesions of endometriosis are better approached by a technique eg. Retrograde hysterectomy (Author's innovation). In this technique, as there is no scope to identify the pedicles from above, we find a clear area near the vault usually anterior, sometime one lateral aspect and proceed from this area step by step - first the vault, then the parametrium \& uterosacral ligaments, uterine vessels, infundibulopelvic ligament and the round ligaments. During the process one has to choose the area in continuity and take care to avoid the vital structures nearby. The author has completed more than 500 cases during last 20 years period with this technique and found encouraging results.

\section{Adhesiolysis in difficult situations}

In cases of endometriosis and malignant infiltration adhesions with neighboring viscera and vessels are quite difficult to handle. Main principle is to stay on uterus and cut in to uterus and to use sharp dissection as much as possible. But in certain delicate cases where the intestines are adhered very closely or a lymphnode is attached to a big vessels it is really dangerous. In such cases, the author has used his innovated technique of hydrodissection which is quite cost effective ${ }^{6}$. For this normal saline is used either by a $10 \mathrm{cc}$ syringe or by a IV drip set with pressure at the site of adhesions which makes adhered tissue softer, then they could easily be separated by little effort without damaging the vital structures.

\section{Conclusion}

A difficult hysterectomy should always be anticipated before hand, planned in a systematic way and needs an experienced surgeon. Different tips of surgery as indicated for both vaginal and abdominal hysterectomy should be followed. Retrograde hysterectomy is an useful technique for difficult cases where the ovarian pedicles, uterine arteries - all are obscured in adhesions. Hydrodissection technique described here is a useful technique, cost effective and without any side effect.

\section{Conflict of interest: None. Disclaimer: Nil.}

\section{References}

1.Whiteman MK, Hills SD, Jamieson DJ, Morrow B, Podgornik MN, Brett KM, et al. Inpatient hysterectomy surveillance in the US, 2000-2004. Am J Obstet Gynaecol. 2008; 198(1); 34.e1-7.

2.Julian TM. Vaginal Hysterectomy: an apparent exception to evidence based decision making. Obstet Gynecol. 2008: 111 (4): 812-3.

3.Gebhart JB, Occhino JA. Difficult vaginal hysterectomy. Clin Obstet Gynecol. 2010 Mar; 53(1): 40-50.

4.Chen CC, Korn A, Klingele C, Barber MD, Paraiso MF, Walters MD, et al. Objective assessment of vaginal skills. Am J. Obstet Gynecol. 2010; 203 (1); 79e1-8

5.Sarma HK. Radical hysterectomy - its trend and role in treatment of carcinoma cervix. The New Indian Journal of OBGYN. 2016 (July-Dec); 3(1): 1-3. 